\title{
ON MATRIX SUMMABILITY OF JACOBI SERIES
}

\author{
SHYAM LAL
}

Abstract. In this paper a new theorem on Matrix Summability of Jacobi series is established.

This theorem is a generalization of several known and unknown results.

\section{Definitions and Notations}

Let $f(x)$ be defined in closed interval $[-1,1]$ such that the function $(1-x)^{\alpha}(1+$ $x)^{\beta} f(x) \in L[-1,1] ; \alpha>-1, \beta>-1$. The Jacobe series corresponding to this function is

$$
f(x)=\sum_{n=0}^{\infty} a_{n} P_{n}^{(\alpha, \beta)}(x)
$$

where

$$
a_{n}=\frac{(2 n+\alpha+\beta+1) \Gamma(n+1) \Gamma(n+\alpha+\beta+1)}{2^{\alpha+\beta+1} \Gamma(n+\alpha+1) \Gamma(n+\beta+1)} \int_{-1}^{1}(1-x)^{\alpha}(1+x)^{\beta} f(x) P_{n}^{(\alpha, \beta)}(x) d x
$$

and $P_{n}^{(\alpha, \beta)}$ are the Jacobi polynomials.

Let $T=\left(a_{n, k}\right)$ be an infinite triangular matrix satisfying the Silverman-Toeplitz (1913) condition of regularity i.e.

$$
\begin{array}{ll}
\sum_{k=0}^{n} a_{n, k} \rightarrow 1 & \text { as } n \rightarrow \infty, \\
a_{n, k}=0 \quad \text { for } k>n
\end{array}
$$

and $\sum_{k=0}^{n}\left|a_{n, k}\right| \leq M$, a finite constant. $\sum_{\nu=0}^{k} u_{\nu}$.

Let $\sum u_{n}$ be an infinite series with the sequence of partial sums $\left\{s_{k}\right\}$ where $s_{k}=$ The sequence-to-sequence transformation

$$
\begin{aligned}
t_{n} & =\sum_{k=0}^{n} a_{n, k} s_{k} \\
& =\sum_{k=0}^{n} a_{n, n-k} s_{n-k}
\end{aligned}
$$

Received November 2, 1998.

Key words and phrases. Analytic, univalent, fractional calculus, Jacobi series, Nörlund summability, Matrix summability. 
defines the sequence $\left\{t_{n}\right\}$ of Matrix means of the sequence $\left\{s_{n}\right\}$, generated by the sequence of coefficients $\left(a_{n, k}\right)$. The series $\sum u_{n}$ is said to be summable to the sum $S$ by Matrix method if $\lim _{n \rightarrow \infty} t_{n}$ exists and is equal to $s$ (Zygmund (1935)) and we write $t_{n} \rightarrow s(T)$, as $n \rightarrow \infty$.

Four important particular cases of matrix means are

(i) harmonic mean, where $a_{n, k}=\frac{1}{(n-k+1) \log n}$

(ii) $(H, p)$ mean, when $a_{n, k}=\frac{1}{(\log )^{p-1}(n+1)} \prod_{q=0}^{p-1} \log ^{q}(k+1)$

(iii) Nörlund mean (1919) when $a_{n, k}=\frac{p_{n-k}}{p_{n}}$ when $P_{n}=\sum_{k=0}^{n} p_{k}$

(iv) Generalized Nörlund mean (1958) where $a_{n, k}=\frac{p_{n-k} q_{k}}{R_{n}}$ where $R_{n}=\sum_{k=0}^{n} p_{k} q_{n-k}$.

We use following notations:

$$
F(\phi)=\{f(\cos \phi)-A\}\left(\sin \frac{\phi}{2}\right)^{2 \alpha+1}\left(\cos \frac{\phi}{2}\right)^{2 \beta+1},
$$

A being fixed constant

$$
\psi(t)=\int_{0}^{t}|F(\phi)| d \phi
$$

$\tau=$ Integral part of $\frac{1}{\phi}=\left[\frac{1}{\phi}\right]$

$$
A_{n, \tau}=\sum_{k=0}^{\tau} a_{n, n-k}
$$

$\eta=$ Integral part of $\frac{1}{\delta}=\left[\frac{1}{\delta}\right]$.

\section{Main Theorem}

The Nörlund summability $\left(N, p_{n}\right)$ of Jacobi series has been studies by a number of researchers like Gupta (1970), Choudhary (1970), Thorpe (1975), Pandey and Beohar (1978), Prasad and Saxena (1979), Beohar and Sharma (1980). Pandey (1981) and Tripathi, Tripathi and Yadav (1988) After quite a good amout of work in the ordinary Nörlund summability of Jacobi series at the point $x=1$, Khare and Tripathi (1988) discussed generalized Nörlund summability $(N, p, q)$ of Jacobe series. $(N, p, q)$ summability reduces to $\left(N, p_{n}\right)$ summability for $q_{n}=1 \quad \forall n$ and to $\left(\bar{N}, q_{n}\right)$ means when $p_{n}=1 \quad \forall n$. But nothing seems to have been done so far in the direction of study of Jacobi series by Matrix summability method which, as known, includes, as special cases, the methods of $\left(N, p_{n}\right)$ and $(N, p, q)$ summabilities. In an attempt to make an advance study in this direction we, in the present paper, establish the following theorem for the Matrix summability of Jacobi series. 
Theorem Let $T=\left(a_{n, k}\right)$ be an infinite triangular regular matrix such that the elements $\left(a_{n, k}\right)$ be non-negative, non-decreasing with $k, n^{(2 \alpha+1) / 2} A_{n, \eta} \rightarrow 0$ as $n \rightarrow \infty$.

$$
\text { If } \psi(t)=\int_{0}^{t}|F(\phi)| d \phi=O\left(\frac{t^{2 \alpha+2}}{\xi\left(\frac{1}{t}\right)}\right) \text {, as } t \rightarrow 0
$$

where $\xi(t)$ be positive, non-decreasing with $t$ such that $\xi(n) \rightarrow \infty$ as $n \rightarrow \infty$ and

$$
\sum_{a}^{n} \frac{A_{n, k}}{k^{(2 \alpha+3)} \xi(k)}=O\left(\frac{1}{n^{(2 \alpha+1) / 2}}\right),
$$

a being a fixed positive integer, then Jacobi series (1.1) is matrix summable $(T)$ at $x=1$ to the sum $A$ provided $-\frac{1}{2} \leq \alpha<\frac{1}{2} ; \beta>-\frac{1}{2}$ and the antipole condition

$$
\int_{-1}^{b}(1+x)^{(2 \beta-3) / 4}|f(x)| d x<\infty
$$

$b$ fixed, is satisfied.

\section{Lemmas}

The following lemmas are required for the proof of the theorem.

Lemma 1.[Szegö(1959)] If $\alpha>-1, \beta>-1$, then as $n \rightarrow \infty$.

$$
\begin{aligned}
P_{n}^{(\alpha, \beta)}(\cos \phi) & =O\left(n^{\alpha}\right), \quad 0 \leq \phi \leq \frac{1}{n} \\
& =O\left(n^{\beta}\right), \quad \pi-\frac{1}{n} \leq \phi \leq \pi \\
& =n^{-1 / 2} k(\phi)\left[\cos (N \phi+\nu)+\frac{O(1)}{n \sin \phi}\right], \quad \frac{1}{n} \leq \phi \leq \pi-\frac{1}{n}
\end{aligned}
$$

where

$$
\begin{aligned}
& k(\phi)=, \quad{ }^{1 / 2}\left(\sin \frac{\phi}{2}\right)^{-\alpha-1 / 2}\left(\cos \frac{\phi}{2}\right)^{-\beta-1 / 2} \\
& N=n+\quad \frac{-\beta+1}{?}, \quad \nu=-\left(\alpha+\frac{1}{2}\right) \frac{\pi}{4} .
\end{aligned}
$$

Lemma 2. [Gupta (1970)] The antipole condition (2.3) implies that

$$
\int_{\delta}^{\pi}\left(\cos \frac{\phi}{2}\right)^{(2 \beta-1) / 2}|f \cdot(\cos \phi)-A| d \phi<\infty
$$

which fruther implies that

$$
\int_{0}^{1 / n} t^{(2 \beta-1) / 2}|f(-\cos t)-A| d t=O(1)
$$


Lemma 3. [McFadden (1942)]: If $\left\{p_{n}\right\}$ is a non-negetive and non-increasing sequence, then for $0 \leq a<b \leq \infty, 0 \leq \phi \leq \pi$ and for any $n$ and $a$,

$$
\left|\sum_{a}^{b} p_{k} e^{i(n-k) \phi}\right|=O\left(P_{\tau}\right)
$$

where $P_{\tau}=P_{(1 / \phi)}$ and $\tau=\left[\frac{1}{\phi}\right]$.

Lemma 4. [Rhoades (1984)]: Let $\left\{u_{n}\right\},\left\{v_{n}\right\}$ be real sequences $\left\{u_{n}\right\}$ non-negative. If $\left\{v_{n}\right\}$ is non-increasing, then

$$
\left|\sum_{k=1}^{n} u_{k} v_{k}\right| \leq v_{1} \max _{1 \leq r \leq n}\left|\sum_{k=1}^{r} u_{k}\right|
$$

If $\left\{v_{n}\right\}$ is non-decreasing, then

$$
\left|\sum_{k=1}^{n} u_{k} v_{k}\right| \leq 2 v_{n} \max _{1 \leq r \leq n}\left|\sum_{k=1}^{r} u_{k}\right|
$$

Lemma 5. Under the condition of the theorem on $\left(a_{n, k}\right)$, for large $n$, uniformly in $0<\phi \leq \pi, 0 \leq a \leq b \leq n$,

$$
\left|\sum_{k=a}^{b} a_{n, n-k} \cos \{(n-k+\rho) \phi-r\}(n-k)^{\alpha+1 / 2}=O\right|\left(n^{\alpha+1 / 2} A_{n, \tau}\right)
$$

where $\rho=\frac{\alpha+\beta+2}{2}, \gamma=-\left(\alpha+\frac{3}{2}\right) \frac{\pi}{4}$.

Proof.

$$
\begin{aligned}
& \left|\sum_{k=a}^{b} a_{n, n-k} \cos \{(n-k+\rho) \phi-r\}(n-k)^{\alpha+1 / 2}\right| \\
= & O\left(n^{\alpha+1 / 2}\right) \mid \text { Real part of } \sum_{k=a}^{b} a_{n, n-k} e^{i\{(n-k+\rho) \phi-r\}} \mid \text {, by Lemma (4) } \\
= & O\left(n^{\alpha+1 / 2}\right)\left[\left|\sum_{k=a}^{b} a_{n, n-k} e^{i(n-k) \phi} e^{i(\rho \phi-r)}\right|\right] \\
= & O\left(n^{\alpha+1 / 2}\right)\left[\left|\sum_{k=a}^{b} a_{n, n-k} e^{i(n-k) \phi}\right|\right] \\
= & O\left(n^{\alpha+1 / 2} A_{n, \tau}\right), \quad \text { by Lemma (3), }
\end{aligned}
$$

which proves the result. 
Lemma 6. Under the hypothesis of the theorem,

$$
\sum_{k=0}^{n-1} a_{n, n-k}(n-k)^{\alpha-\frac{1}{2}}=O\left(n^{\alpha-1 / 2}\right)
$$

Proof.

$$
\begin{aligned}
\sum_{k=0}^{n-1} a_{n, n-k}(n-k)^{\alpha-\frac{1}{2}} & =O\left(n^{\alpha-\frac{1}{2}}\right)\left[\sum_{k=0}^{n-1} a_{n, n-k}\right] \text { by Lemma } 4 \\
& =O\left(n^{\alpha-\frac{1}{2}}\right)\left[\sum_{k=0}^{n} a_{n, n-k}\right]=O\left(n^{\left.\alpha-\frac{1}{2}\right)} A_{n, n}\right. \\
& =O\left(n^{\alpha-1 / 2}\right) O(1) \\
& =O\left(n^{\alpha-1 / 2}\right),
\end{aligned}
$$

which proves the result.

Lemma 7. Let

$$
M_{n}(\phi)=2^{\alpha+\beta+1} \sum_{k=0}^{n-1} a_{n, n-k} \lambda_{n-k} p_{n-k}^{(\alpha+1, \beta)}(\cos \phi)
$$

where

$$
\lambda_{n}=\frac{2^{-(\alpha+\beta+1)} \Gamma(n+\alpha+\beta+2)}{\Gamma(\alpha+1) \Gamma(n+\beta+1)} \cong \frac{2^{-(\alpha+\beta+1)}}{\Gamma(\alpha+1)} n^{\alpha+1}
$$

then, for $\frac{1}{2}>\alpha \geq-\frac{1}{2}, \beta>-\frac{1}{2}$ and if $\left(a_{n, k}\right)$ satisfied the hypothesis of the theorem,

$$
\begin{aligned}
M(\phi)= & O\left(n^{2 \alpha+2}\right) \quad \text { if } 0 \leq \phi<\frac{1}{n} \\
= & O\left(n^{\alpha+\beta+1}\right) \quad \text { if } \pi-\frac{1}{n} \leq \phi \leq \pi \\
= & O\left[n^{\alpha+1 / 2} A_{n, \tau}\left(\sin \frac{\phi}{2}\right)^{-\alpha-3 / 2}\left(\cos \frac{\phi}{2}\right)^{-\beta-1 / 2}\right] \\
& +O\left[n^{\alpha-1 / 2}\left(\sin \frac{\phi}{2}\right)^{-\alpha-5 / 2}\left(\cos \frac{\phi}{2}\right)^{-\beta-1 / 2}\right] \quad \frac{1}{n} \leq \phi \leq \pi-\frac{1}{n}
\end{aligned}
$$

Proof. For $0 \leq \phi \leq \frac{1}{n}$

$$
\begin{aligned}
M_{n}(\phi) & =O\left(2^{\alpha+\beta+1}\right) \sum_{k=0}^{n-1}\left[a_{n, n-k} O\left(2^{-(\alpha+\beta+1)}\right)(n-k)^{\alpha+1}(n-k)^{\alpha+1}\right] \\
& =O(1)\left[\sum_{k=0}^{n-1} a_{n, n-k} O(n-k)^{2 \alpha+2}\right] \quad \text { by }(3.1)
\end{aligned}
$$




$$
\begin{aligned}
& =O\left(n^{2 \alpha+2}\right) \sum_{k=0}^{n-1} a_{n, n-k}, \quad \text { by Lemma (4) } \\
& =O\left(n^{2 \alpha+2}\right) \sum_{k=0}^{n} a_{n, n-k}=O\left(n^{2 \alpha+2}\right)\left(A_{n, n}\right) \\
& =O\left(n^{2 \alpha+2}\right) O(1) \\
& =O\left(n^{2 \alpha+2}\right)
\end{aligned}
$$

If $\pi-\frac{1}{n} \leq \phi \leq \pi$, using (3.2), we have

$$
\begin{aligned}
M_{n}(\phi) & =O\left[\sum_{k=0}^{n-1} a_{n, n-k} O(n-k)^{\beta} O(n-k)^{\alpha+1}\right] \\
& =O\left[\sum_{k=0}^{n-1} a_{n, n-k} O(n-k)^{\alpha+\beta+1}\right] \\
& =O\left(n^{\alpha+\beta+1}\right) \sum_{k=0}^{n-1} a_{n, n-k}, \quad \text { by Lemma (4) } \\
& =O\left(n^{\alpha+\beta+1}\right) \sum_{k=0}^{n} a_{n, n-k}=O\left(n^{\alpha+\beta+1}\right) A_{n, n} \\
& =O\left(n^{\alpha+\beta+1}\right) O(1) \\
& =O\left(n^{\alpha+\beta+1}\right)
\end{aligned}
$$

If $\frac{1}{n} \leq \phi \leq \pi-\frac{1}{n}$, we have, with notation as in Lemma 5 .

$$
\begin{aligned}
M_{n}(\phi)= & O(1) \sum_{k=0}^{n-1}\left[a_{n, n-k}(n-k)^{\alpha+1 / 2}\left(\sin \frac{\phi}{2}\right)^{-\alpha-3 / 2}\left(\cos \frac{\phi}{2}\right)^{-\beta-1 / 2}\right. \\
& \left.\cdot \cos \{(n-k) \phi+\rho \phi-r\}+\frac{O(1)}{(n-k) \sin \phi}\right], \text { by }(3.3) \\
= & O(1)\left[\sum_{k=0}^{n-1} a_{n, n-k}(n-k)^{\alpha+1 / 2}\left(\sin \frac{\phi}{2}\right)^{-\alpha-3 / 2}\left(\cos \frac{\phi}{2}\right)^{-\beta-1 / 2}\right. \\
& \cos \{(n-k) \phi+\rho \phi-r\}] \\
+ & O(1)\left[\sum_{k=0}^{n-1} a_{n, n-k}(n-k)^{\alpha-1 / 2}\left(\sin \frac{\phi}{2}\right)^{-\alpha-5 / 2}\left(\cos \frac{\phi}{2}\right)^{-\beta-3 / 2}\right] \\
= & O(1)\left[\sum_{k=0}^{n-1} a_{n, n-k}\left(\sin \frac{\phi}{2}\right)^{-\alpha-3 / 2}\left(\cos \frac{\phi}{2}\right)^{-\beta-1 / 2}\right. \\
& \left.(n-k)^{\alpha+1 / 2} \cos \{(n-k) \phi+\rho \phi-r\}\right]
\end{aligned}
$$




$$
\begin{aligned}
& +O\left(n^{\alpha-1 / 2}\right)\left[\sum_{k=0}^{n-1} a_{n, n-k}\left(\sin \frac{\phi}{2}\right)^{-\alpha-5 / 2}\left(\cos \frac{\phi}{2}\right)^{-\beta-3 / 2}\right] \text { by Lemma } 4 \\
= & O\left(\left(\sin \frac{\phi}{2}\right)^{-\alpha-3 / 2}\left(\cos \frac{\phi}{2}\right)^{-\beta-1 / 2}\right) \\
& {\left[\sum_{k=0}^{n-1} a_{n, n-k}(n-k)^{\alpha+1 / 2} \cos \{(n-k) \phi+\rho \phi-r\}\right] } \\
& +O\left(\left(\sin \frac{\phi}{2}\right)^{-\alpha-5 / 2}\left(\cos \frac{\phi}{2}\right)^{-\beta-3 / 2}\right)\left[\sum_{k=0}^{n-1} a_{n, n-k}(n-k)^{\alpha-1 / 2}\right] \\
= & O\left(\left(\sin \frac{\phi}{2}\right)^{-\alpha-3 / 2}\left(\cos \frac{\phi}{2}\right)^{-\beta-1 / 2}\right) O\left(n^{\alpha+1 / 2} A_{n, \tau}\right) \\
& +O\left(\left(\sin \frac{\phi}{2}\right)^{-\alpha-5 / 2}\left(\cos \frac{\phi}{2}\right)^{-\beta-3 / 2}\right) O\left(n^{\alpha-1 / 2}\right) \text { by Lemma (5) and (6) } \\
= & O\left[n^{\alpha+1 / 2} A_{n, \tau}\left(\sin \frac{\phi}{2}\right)^{-\alpha-3 / 2}\left(\cos \frac{\phi}{2}\right)^{-\beta-1 / 2}\right] \\
& +O\left[n^{\alpha-1 / 2}\left(\sin \frac{\phi}{2}\right)^{-\alpha-5 / 2}\left(\cos \frac{\phi}{2}\right)^{-\beta-3 / 2}\right]
\end{aligned}
$$

In this way Lemma (7) is proved.

\section{Proof of the Theorem.}

Following Obrechkoff (1936) the $n$th partial sum of the series (1.1) at the point $x=1$ is given by

$$
S_{n}(1)=2^{\alpha+\beta+1} \int_{0}^{\pi}\left(\sin \frac{\phi}{2}\right)^{2 \alpha+1}\left(\cos \frac{\phi}{2}\right)^{2 \beta+1} f(\cos \phi) S_{n}^{\prime}(1, \cos \phi) d \phi
$$

where $S_{n}^{\prime}(1, \cos \phi)$ denotes the $n$th partial sum of the series

$$
\sum_{m} \frac{P_{m}^{(\alpha, \beta)}(1) P_{m}^{(\alpha, \beta)}(\cos \phi)}{g_{m}}
$$

where $g_{m}=\frac{(2 n+\alpha+\beta+1) \sqrt{(n+1)} \sqrt{(n+\alpha+\beta+1)}}{2^{\alpha+\beta+1} \sqrt{(n+\alpha+1)} \sqrt{(n+\beta+1)}}$

Rao (1929) has shown that

$$
S_{n}^{\prime}(1, \cos \phi)=\lambda_{n} P_{n}^{(\alpha+1, \beta)} \cos (\phi) .
$$

Therefore

$$
\begin{aligned}
S_{n}(1)-A & =2^{\alpha+\beta+1} \lambda_{n} \int_{0}^{\pi}\left(\sin \frac{\phi}{2}\right)^{2 \alpha+1}\left(\cos \frac{\phi}{2}\right)^{2 \beta+1}\left\{f(\cos \phi-A\} P_{n}^{(\alpha+1, \beta)}(\cos \phi) d \phi\right. \\
& =2^{\alpha+\beta+1} \lambda_{n} \int_{0}^{\pi} F(\phi) P_{n}^{(\alpha+1, \beta)}(\cos \phi) d \phi,
\end{aligned}
$$


where $\lambda_{n}$ is defined as in Lemma (7).

The matrix means of the series (1.1) at $x=1$ is given by

$$
\begin{aligned}
t_{n} & =\sum_{k=0}^{n} a_{n, k} S_{k}(1) \\
& =\sum_{k=0}^{n} a_{n, n-k} S_{n-k}(1) \\
\text { or } t_{n}-A & =\sum_{k=0}^{n} a_{n, k}\left\{S_{n-k}(1)-A\right\} \\
& =\int_{0}^{\pi} F(\phi) M_{n}(\phi) d \phi+\left(a_{n, 0}\right) O(1) \int_{0}^{\pi} F(\phi) d \phi .
\end{aligned}
$$

Since $\int_{0}^{\pi} F(\phi) d \phi$ is a finite constant, by assumption, second term on the right is $O(1)$ as $n \rightarrow \infty$. Hence in order to prove theorem we have to show that

$$
I=\int_{0}^{\pi} F(\phi) N(\phi) d \phi=O(1) \text { as } n \rightarrow \infty
$$

Let us denote

$$
\begin{aligned}
I & =\left[\int_{0}^{1 / n}+\int_{1 / n}^{\delta} \int_{\delta}^{\pi-1 / n}+\int_{\pi-1 / n}^{\pi}\right] F(\phi) M_{n}(\phi) d \phi \\
& =I_{1}+I_{2}+I_{3}+I_{4}, \quad \text { say }
\end{aligned}
$$

$\delta$ being a suitable constant.

$$
\begin{aligned}
I_{1}= & \int_{0}^{1 / n} F(\phi) M_{n}(\phi) d \phi \\
\left|I_{1}\right| & =\int_{0}^{1 / n}|F(\phi)| O\left(n^{2 \alpha+2}\right) d \phi, \quad \text { by }(3.11) \\
& =O\left(n^{2 \alpha+2}\right) \int_{0}^{1 / n}|F(\phi)| d \phi \\
& =O\left(n^{2 \alpha+2}\right) o\left(\frac{1}{n^{2 \alpha+2} \beta(n)}\right), \quad \text { by }(2.1) \\
& =o\left(\frac{1}{\xi(n)}\right) \\
& =o(1), \text { as } n \rightarrow \infty, \text { by hypothesis of theorem } .
\end{aligned}
$$

In order to estimate $I_{2}$ we employ the asymptotic relation given in (3.13). Thus

$$
I_{2}=O\left[\int_{1 / n}^{\delta}|F(\phi)| n^{(2 \alpha+1) / 2} A_{n, \tau}\left(\sin \frac{\phi}{2}\right)^{-(2 \alpha+3) / 2} d \phi\right]
$$




$$
\begin{aligned}
& +O\left[\int_{1 / n}^{\delta}|F(\phi)| n^{(2 \alpha+1) / 2}\left(\sin \frac{\phi}{2}\right)^{-(2 \alpha+5) / 2} d \phi\right] \\
= & I_{2.1}+I_{2.2}, \quad \text { say }
\end{aligned}
$$

Now

$$
\begin{aligned}
& I_{2.1}=O\left(n^{(2 \alpha+1) / 2}\right)\left[\int_{1 / n}^{\delta} \frac{|F(\phi)| A_{n, \tau} d \phi}{\phi^{(2 \alpha+3) / 2}}\right] \\
& =O\left(n^{(2 \alpha+1) / 2}\right)\left[o\left(\frac{\phi^{2 \alpha+2}}{\xi\left(\frac{1}{\phi}\right)} \frac{A_{n, \tau}}{\phi^{(2 \alpha+3) / 2}}\right)_{1 / n}^{\delta}+o\left\{\int_{1 / n}^{\delta} \frac{\phi^{2 \alpha+2}}{\xi\left(\frac{1}{\phi}\right)} \frac{d}{d \phi}\left(\frac{A_{n, \tau}}{\phi^{(2 \alpha+3) / 2}}\right) d \phi\right]\right. \\
& =o\left(\frac{A_{n, n}}{\xi(n)}+o\left(n^{(2 \alpha+1) / 2} A_{n, \eta}\right)\right. \\
& +o\left(n^{(2 \alpha+1) / 2}\right) \int_{1 / n}^{\delta} \frac{\phi^{2 \alpha+2}}{\xi\left(\frac{1}{\phi}\right)} \frac{A_{n, \tau}}{\phi^{(2 \alpha+5) / 2}} d \phi \\
& =o(1)+o(1)+o\left(n^{(2 \alpha+1) / 2}\right) \int_{1 / \delta}^{n} \frac{A_{n, u}}{u^{(2 \alpha+3) / 2} \xi(u)} d u, \\
& \frac{1}{\phi}=u \text { by the hypothesis of theorem } \\
& =o(1)+o\left(n^{(2 \alpha+1) / 2}\right) \sum_{a}^{n} \frac{A_{n, k}}{\xi(k) k^{(2 \alpha+3) / 2}} \text { where } a=\left[\frac{1}{\delta}\right]+1, \quad n \geq\left[\frac{1}{t}\right] \\
& =o(1), \quad \text { by }(2.2) \text {. } \\
& I_{2.2}=O\left[\int_{1 / n}^{\delta}|F(\phi)| n^{(2 \alpha-1) / 2}\left(\sin \frac{1}{2} \phi\right)^{-(2 \alpha+5) / 2} d \phi\right] \\
& =O\left(n^{(2 \alpha-1) / 2}\right)\left[\int_{1 / n}^{\delta} \frac{F(\phi)}{\phi^{(2 \alpha+5) / 2}} d \phi\right] \\
& =O\left(n^{(2 \alpha-1) / 2}\right)\left[\left\{\frac{1}{\phi^{(2 \alpha+5) / 2}} o\left(\frac{\phi^{2 \alpha+2}}{\xi\left(\frac{1}{\phi}\right)}\right)\right\}_{1 / n}^{\delta}+o\left\{\int_{1 / n}^{\delta} \frac{\phi^{(2 \alpha-3) / 2}}{\xi\left(\frac{1}{\phi}\right)} d \phi\right\}\right] \\
& =o\left(n^{(2 \alpha-1) / 2}\right)+o\left(n^{(2 \alpha-1) / 2}\right) o\left(\frac{n^{-(2 \alpha-1) / 2}}{\xi(n)}\right)+o\left(n^{(2 \alpha-1) / 2}\right) \int_{1 / n}^{\delta} \frac{\phi^{(2 \alpha-3) / 2}}{\xi\left(\frac{1}{\phi}\right)} d \phi \\
& =o\left(n^{(2 \alpha-1) / 2}\right)+o\left(\frac{1}{\xi(n)}\right)+o\left(\frac{n^{(2 \alpha-1) / 2}}{\xi(n)}\right) \int_{1 / n}^{\delta} \phi^{(2 \alpha-3) / 2} d \phi \text {, by mean value theorem } \\
& =o(1)+o\left(\frac{n^{(2 \alpha-1) / 2}}{\xi(n)}\right)\left\{\frac{\phi^{(2 \alpha-1) / 2}}{(2 \alpha-1) / 2}\right\}_{1 / n}^{\delta} \quad\left(\because-\frac{1}{2} \leq \alpha<\frac{1}{2}\right) \\
& =o(1)+o\left(\frac{n^{(2 \alpha-1) / 2}}{\xi(n)}\right)+o\left(\frac{n^{(2 \alpha-1) / 2}}{\xi(n)}\right)\left(n^{-(2 \alpha-1) / 2}\right)
\end{aligned}
$$




$$
\begin{aligned}
& =o(1)+\left(\frac{n^{(2 \alpha-1) / 2}}{\xi(n)}\right)+o\left(\frac{1}{\xi(n)}\right) \\
& =o(1)+o(1) \\
& =o(1), \quad \text { as } n \rightarrow \infty .
\end{aligned}
$$

Considering $I_{3}$, we have

$$
\begin{aligned}
I_{3}= & O\left[\int_{\delta}^{\pi-1 / n} \frac{|F(\phi)| A_{n, \tau} \cdot n^{(2 \alpha+1) / 2}}{\left(\sin \frac{\phi}{2}\right)^{(2 \alpha+3) / 2}\left(\cos \frac{\phi}{2}\right)^{(2 \beta+1) / 2}} d \phi\right] \\
& +O\left(n^{(2 \alpha-1) / 2}\right)\left[\int_{\delta}^{\pi-1 / n}|F(\phi)| \frac{d \phi}{\left(\sin \frac{\phi}{2}\right)^{(2 \alpha+5) / 2}\left(\cos \frac{\phi}{2}\right)^{(2 \beta+3) / 2}}\right] \\
= & O\left(n^{(2 \alpha+1) / 2} A_{n . \eta}\right) \int_{\delta}^{\pi-1 / n}|F(\cos \phi)-A|\left(\cos \frac{\phi}{2}\right)^{(2 \beta-1) / 2} \cos \frac{\phi}{2} d \phi \\
& +O\left(n^{(2 \alpha-1) / 2}\right) \int_{\delta}^{\pi-1 / n}|F(\cos \phi)-A|\left(\cos \frac{\phi}{2}\right)^{(2 \beta-1) / 2} d \phi \\
= & O\left(n^{(2 \alpha+1) / 2} A_{n, \eta}\right)+O\left(n^{(2 \alpha-1) / 2}\right), \quad \text { by }(3.4) \\
= & O(1)+o(1), \quad \text { as } n \rightarrow \infty \\
= & o(1) . \quad \text { as } n \rightarrow \infty .
\end{aligned}
$$

We finally consider $I_{4}$.

$$
\begin{aligned}
I_{4} & =O\left(n^{\alpha+\beta+1}\right) \int_{\pi-1 / n}^{\pi}|F(\phi)| d \phi, \quad \text { by }(3.12) \\
& =O\left(n^{\alpha+\beta+1}\right) \int_{\pi-1 / n}^{\pi}|f(\cos \phi)-A|\left(\sin \frac{\phi}{2}\right)^{2 \alpha+1}\left(\cos \frac{\phi}{2}\right)^{2 \beta+1} d \phi \\
& =O\left(n^{a+\beta+1}\right) \int_{0}^{1 / n}|f(-\cos t)-A|\left(\cos \frac{t}{2}\right)^{2 \alpha+1}\left(\sin \frac{t}{2}\right)^{2 \beta+1} d t \quad \text { taking } \pi-\phi=t \\
& =O\left(n^{\alpha+\beta+1}\right) \int_{0}^{1 / n}|f(-\cos t)-A| t^{2 \beta+1} \mathrm{dt} \\
& =O\left(n^{2 \alpha-1 / 2}\right) \int_{0}^{1 / n}|f(-\cos t)-A| t^{(2 \beta-1) / 2} \mathrm{dt} \\
& =O(1), \quad \text { as } n \rightarrow \infty . \quad \text { by }(3.5)
\end{aligned}
$$

Thus the theorem is completely established.

\section{Particular Cases}

(a) If $a_{n, k}=\frac{p_{n-k}}{P_{n}}$ and $\xi(x)=\log x$, result of Gupta (1970) becomes the particular case of main theorem. 
(b) Result of Tripathi, Tripathi and Yadav (1988) becomes the particular case of our theorem if $\left(a_{n, k}\right)$ is defined as in (a) and $\xi(x)=\left(P_{[x]}\right)^{c} ; 0<c<1$.

(c) If $a_{n, k}=\frac{p_{n-k} q_{n}}{R_{n}}$ where $R_{n}=\sum_{k=0}^{n} p_{k} q_{n-k}$ and $\xi(x)$ is as defined as in (a) then result of Khare and Tripathi (1988) becomes the particular case of main theorem.

\section{Acknowledgement}

The author is grateful to his teacher Prof. L. M. Tripathi for suggesting the problem and to Prof. S. N. Lal, Head, Department of Mathematics, Banaras Hindu University, Varanasi (India) who has taken the pain to see the manuscript.

\section{References}

[1] B. K. Beohar and K. G. Sharma, On Nörlund summability of Jacobi series, Indian J. Pure Appl. Maths., 11(1980), 1475-1481.

[2] D. Borwein, On product of sequences, Jour. London Math. Soc., 33(1958), 352-357.

[3] R. S. Choudhary, On Nörlund summability of Jacobi series, Rend. Ace. Naz. Lincei, Ser., . 52(1970), 644-352.(?)

[4] D. P. Gupta, Nörlund summability of Jacobi series, D. Sc. Thesis, University of Allahabad, Allahabad (1970).

[5] G. H. Hardy, Divergent series, The University Press, Oxford, (1949).

[6] S. P. Khare and S. K. Tripathi, On $(N, p, q)$ summability of Jacobi series, Indian J. Pure Appl. Math., 19(1980), 353-368.

[7] L. McFadden, Absolute Nörlund summability, Duke Math. J., 9(1942), 168-207.

[8] N. E. Nörlund, Surune application des fonctions permutables, Lund. Universitets Arsskrift, 16(1919), 1-10.

[9] N. Obrechkoff, Formules asymototiques pour les polynomes des Jacobi et surles series suivant les memes polynomes (Russian), Ann. Univ. Sofia Foc. Phys. Math., 32(1939), $39-135$.

[10] B. N. Pandey, On the study of Jacobe series by $\left(N, p_{n}\right)$-method, Indian J. Pure Appl. Math., 12(1981), 1438-1447.

[11] G. S. Pandey and B. K. Beohar, On Nörlund summability of Jacobi series, Indian J. Pure Appl. Math., 9(1978), 501-509.

[12] Rajendra Prasad and Ashok Saxena, On the Nörlund summability of Fourier-Jacobi series, Indian J. Pure Appl. Math., 10(1979), 1303-1311.

[13] H. Rao, Über die Lebesgueschen Konstanten der Riehenewick tungennach Jacobischen polynomes, J. Reine Angew. Math., 16(1929), 237-254.

[14] B. E. Rhoedas, Analysis 4,(1984), 323-334.

[15] G. Szegö, Orthogonal Polynomials Colloq. Amer. Math. Soc. Publication, New York. (1959).

[16] L. M. Tripathi, V. N. Tripathi and S. J. Yadav, On Nörlund summability of Jacobi series, Proc. Math. Soc., B. H. U., 4(1988), 183-193.

[17] B. Thorpe, Nörlund summability of Jacobi series, J. Reine Angew. Math., 276(1975), 137-141. 
[18] O. Toeplitz, Überallgemeine lineare Mittelbildungen, P. M. F., 22(1913), 113-119.

[19] A. Zygmund, Trigonometric series, V. Warszawa, (1935), 40-41.

Department of Mathematics \& Statistics Harishchandra Post Graduate College VARANASI221001 (INDIA) 